\title{
Land Resource Inventory and Soil Mapping for Fertility Status of Humnabad Sub-Watershed
}

\author{
C. Thejaswini ${ }^{1 *}$, Ashok S. Alur ${ }^{2}$, M. Shivanna ${ }^{1}$, S. Anil Kumar ${ }^{1}$, S.J. Prashanth ${ }^{1}$, \\ B.N. Dhannanjaya ${ }^{3}$ and Praveen Kumar B. Naikodi ${ }^{4}$ \\ ${ }^{1}$ College of Horticulture, Bengaluru- 560 065, Karnataka, India \\ ${ }^{2}$ University of Horticultural Sciences, Bagalkot -587 104, Karnataka, India \\ ${ }^{3}$ College of Horticulture, Kolar- 517 247, Karnataka, India \\ ${ }^{4}$ College of Horticulture, Bidar- 585 401, Karnataka, India \\ *Corresponding author
}

\begin{abstract}
A B S T R A C T
Growing population pressure and human activities increases the demand on the limited land resources, both for agricultural and other land uses. To meet this unprecedented demand of land for various pursuits, it is imperative to develop land use plans which can

\begin{tabular}{|l|}
\hline Keywords \\
Land resource \\
inventory
\end{tabular}
counteract the detrimental effects on environment and at the same time improve productivity of land. The present paper on land resource inventory and soil mapping for fertility status of Humnabad sub-watershed of Humnabad taluk, Bidar district, Karnataka primarily deals with land resource inventory, soil mapping, their problems and potentials. Cadastral map, Google earth images, toposheets and satellite imagery were used as base maps. Detailed survey was carried out through the study area (1:7920). Surface soil samples were collected at regular grid interval of $360 \mathrm{~cm}$. The collected samples were analyzed in lab for determining their fertility status. Thematic maps were generated using aforementioned satellite data coupled with ground truth. Based on all these fertility status of the sub-watershed was determined. The available nitrogen status of the watershed ranged from low to medium. The available phosphorus and potassium ranged from low to medium. The available sulphur status ranged from low to high but most of the area was low to medium. All the micronutrients were sufficient in lateritic soil series while, in basaltic soil series iron and zinc were low.
\end{abstract}

\section{Introduction}

Indiscriminate use of finite soil resources coupled with lack of management has led to degradation causing concern to planners, researchers and farmers. This calls for a scientific approach for development and management of these resources at various levels. Soil resource inventory provides an insight into potentialities and limitations for its effective management.

It also provides adequate information in terms of land form, natural vegetation as well as characteristics of soils which can be utilized for land resources management and development (Manchanda et al., 2002). Rational utilization of land resources can be 
achieved by optimizing its use, which demands evaluation of land for alternative land use.

\section{Materials and Methods}

The study area, Humnabad sub-watershed, Humnabad taluk, Bidar district, Karnataka. It covers an area of $4880.65 \mathrm{ha}$ and lies between longitude $17^{0} 47^{\prime} 4.218^{\prime \prime}$ to $17^{0} 43$ '23.067" N and latitude $77^{0} 3$ ' 48.24 " to $77^{0} 10^{\prime} 24.99$ ' E. Humnabad sub-watershed covers eight microwatersheds viz., Humnabad, Basawapur, Basavtirth-1, Basavtirth-2, Sadlapur-1, Sadlapur-2, Humnabad west-1 and Humnabad west-2. The average elevation of the district is $520 \mathrm{~m}$ above MSL. Using base maps, Google earth images, satellite imagery and toposheets traversing was done for physiographic delineations in the study area. The site characteristics includes latitude, physiographic-unit, geology, parent material, rainfall, temperature, topography, elevation, erosion and rockiness in the study area were mapped. Land use/land cover mapping was carried out by visual interpretation techniques as per the land use land cover classification system developed by department of space (Anonymous, 1994).

The satellite imageries, LISS- IV were visually interpreted for land use or land cover, were used as reference materials. The soil mapping of Humnabad sub-watershed was done with hydro-geo-morphology, slope, drainage based on tone, texture, size, association etc. The ancillary data namely Survey of India (SOI) topographical sheets on 1:50,000 scale and cadastral map support of remote sensing satellite images adopting visual interpretation procedure on 1:7920 scale. Soil profile studies, soil correlation and classification were carried out by using standard procedures. Surface samples were collected at a regular grid interval of $320 \mathrm{~m}$. Eighteen soil pedons representing the study area were selected based on the topography from various physiographic units identified in the area by field survey. The soils of the study area were categorized into red and black soils based on the geology. Both lateritic and basaltic soil series were found in the study area. The colour of the lateritic soils was dark reddish brown and in case of basaltic soil it varied from dark brown to very dark grayish brown. The soils were described in the field for their morphological properties and later characterized in the laboratory for their physical and chemical properties in the subwatershed. Based on this soil fertility and thematic maps like slope, drainage, land use/land cover, soil, texture, etc. on the scale of 1:7920 scale were generated.

\section{Results and Discussion}

The soil reaction of pedons varied from neutral to slightly alkaline and varied from (SWA) 6.55 to 8.58. Among the lateritic soil series the lowest value (6.55) observed in Karanja Khurd series (KKUiB2G2P2) and ranged from 6.32 to 6.85 , whereas the highest value (7.27) observed in Bhadrapur series (BDPiC3G1) and ranged from 7.16 to 7.38. Among the basaltic series the lowest value (6.56) observed in Dhupat Mahagaon series (DMNmB2G1) and ranged from 6.49 to 6.68 . Whereas, the highest value (8.40) observed in Kouta -B series (KBBmB2) and ranged from 7.86 to 8.99. Soil $\mathrm{pH}$ and EC of major soil pedons increased with depth due to accumulation of leached bases in the subsurface horizons (Table 1). In different soil series organic carbon content ranged from (SWA) 0.41 to 0.78 per cent In general, the surface soil horizons recorded higher organic carbon content than underlying layers and decreased with depth in all soil pedons. The free calcium carbonate content increased with depth. The exchangeable bases were in the order of $\mathrm{Ca}^{2+}>\mathrm{Mg}^{2+}>\mathrm{Na}^{+}>\mathrm{K}^{+}$on the exchangeable complex. 
Table.1 Chemical properties of soil series of Humnabad sub-watershed

\begin{tabular}{|c|c|c|c|c|c|c|}
\hline Mapping unit & Horizon & Depth $(\mathrm{cm})$ & $\mathrm{pH}$ & $\begin{array}{l}\mathrm{EC} \\
\left(\mathrm{dS} \mathrm{m} \mathrm{m}^{-1}\right)\end{array}$ & $\begin{array}{l}\mathrm{OC} \\
(\%)\end{array}$ & $\begin{array}{l}\text { Free } \mathrm{CaCO}_{3} \\
(\%)\end{array}$ \\
\hline \multicolumn{7}{|c|}{ Bhadrapur series } \\
\hline \multirow[t]{2}{*}{ BDPiC3G1 } & Ap & $0-6$ & 7.38 & 0.04 & 0.77 & 0.78 \\
\hline & $\mathrm{Bw}$ & $6-20$ & 7.16 & 0.18 & 0.54 & 0.95 \\
\hline SWA & & & 7.27 & 0.11 & 0.65 & 0.86 \\
\hline \multicolumn{7}{|c|}{ Rampur series } \\
\hline \multirow[t]{2}{*}{ RMPiC3G2P1 } & Ap & $0-6$ & 6.84 & 0.22 & 0.78 & 1.85 \\
\hline & $\mathrm{Bw}$ & $6-20$ & 6.98 & 0.06 & 0.49 & 1.90 \\
\hline SWA & & & 6.91 & 0.14 & 0.63 & 1.87 \\
\hline \multicolumn{7}{|c|}{ Kadambal series } \\
\hline \multirow[t]{3}{*}{ KDMiB2G2P1 } & Ap & $0-8$ & 7.25 & 0.08 & 0.76 & 1.02 \\
\hline & Bt1 & $8-28$ & 7.10 & 0.12 & 0.51 & 1.42 \\
\hline & $\mathrm{Bt} 2$ & $28-40$ & 7.32 & 0.07 & 0.33 & 1.68 \\
\hline SWA & & & 7.22 & 0.09 & 0.53 & 1.37 \\
\hline \multicolumn{7}{|c|}{ Madargi series } \\
\hline \multirow[t]{3}{*}{ MDGiB2G1 } & Ap & $0-10$ & 6.49 & 0.05 & 0.63 & 0.23 \\
\hline & Bt1 & $10-30$ & 6.63 & 0.06 & 0.59 & 0.33 \\
\hline & $\mathrm{Bt} 2$ & $30-50$ & 6.80 & 0.03 & 0.25 & 0.48 \\
\hline SWA & & & 6.64 & 0.04 & 0.49 & 0.34 \\
\hline \multicolumn{7}{|c|}{ Musthari series } \\
\hline \multirow[t]{3}{*}{ MSTiB2G1P1S1 } & Ap & $0-9$ & 6.69 & 0.07 & 0.66 & 0.17 \\
\hline & Bw1 & $9-30$ & 6.70 & 0.08 & 0.48 & 0.25 \\
\hline & Bw2 & $30-60$ & 7.05 & 0.15 & 0.44 & 0.75 \\
\hline SWA & & & 6.81 & 0.07 & 0.52 & 0.39 \\
\hline \multicolumn{7}{|c|}{ Karanja Khurd series } \\
\hline \multirow[t]{3}{*}{ KKUiB2G2P2 } & Ap & $0-10$ & 6.32 & 0.05 & 0.59 & 1.50 \\
\hline & Bw1 & $10-30$ & 6.49 & 0.09 & 0.48 & 1.75 \\
\hline & $\mathrm{Bw} 2$ & $30-70$ & 6.85 & 0.06 & 0.44 & 1.95 \\
\hline SWA & & & 6.55 & 0.06 & 0.50 & 1.73 \\
\hline \multicolumn{7}{|c|}{ Muthangi series } \\
\hline \multirow[t]{4}{*}{ MTNiB2G1 } & Ap & $0-9$ & 7.17 & 0.04 & 0.77 & 0.75 \\
\hline & $\mathrm{Bw}$ & $9-25$ & 7.26 & 0.06 & 0.59 & 1.25 \\
\hline & Bt1 & $25-60$ & 7.06 & 0.08 & 0.55 & 1.55 \\
\hline & $\mathrm{Bt} 3$ & $60-98$ & 7.04 & 0.14 & 0.40 & 1.78 \\
\hline SWA & & & 7.13 & 0.06 & 0.57 & 1.33 \\
\hline \multicolumn{7}{|c|}{ Devgiri series } \\
\hline \multirow[t]{5}{*}{ DGRiB2G1P1 } & Ap & $0-8$ & 6.78 & 0.08 & 0.87 & 0.25 \\
\hline & Bw1 & $8-30$ & 6.96 & 0.1 & 0.85 & 0.75 \\
\hline & Bw2 & $30-60$ & 6.89 & 0.12 & 0.82 & 0.96 \\
\hline & Bw3 & $60-85$ & 7.52 & 0.19 & 0.78 & 1.05 \\
\hline & Bw4 & $85-120$ & 7.54 & 0.18 & 0.51 & 1.15 \\
\hline SWA & & & 7.13 & 0.13 & 0.76 & 0.83 \\
\hline \multicolumn{7}{|c|}{ Mustarwadi series } \\
\hline \multirow[t]{6}{*}{ MWDiB2 } & Ap & $0-8$ & 7.62 & 0.16 & 0.81 & 1.15 \\
\hline & $\mathrm{Bw}$ & $88-39$ & 7.93 & 0.12 & 0.70 & 1.25 \\
\hline & Bt1 & $39-76$ & 6.81 & 0.08 & 0.59 & 1.33 \\
\hline & $\mathrm{Bt} 2$ & $76-98$ & 6.75 & 0.12 & 0.48 & 1.48 \\
\hline & Bt3 & $98-140$ & 6.52 & 0.16 & 0.40 & 1.89 \\
\hline & $\mathrm{Bt} 4$ & $140+$ & 6.40 & 0.21 & 0.29 & 1.97 \\
\hline SWA & & & 7.00 & 0.14 & 0.54 & 1.51 \\
\hline \multicolumn{7}{|c|}{ Basaltic soil series } \\
\hline & & & Lingi seri & & & \\
\hline LINiD3G2P2S4 & Ap & $0-10$ & 7.40 & 0.12 & 0.78 & 10.21 \\
\hline
\end{tabular}


Int.J.Curr.Microbiol.App.Sci (2019) 8(2): 3264-3273

\begin{tabular}{|c|c|c|c|c|c|c|}
\hline & $\mathrm{Bw}$ & $10-21$ & 7.56 & 0.26 & 0.65 & 10.75 \\
\hline SWA & & & 7.48 & 0.19 & 0.71 & 10.48 \\
\hline \multicolumn{7}{|c|}{ Mustapurseries } \\
\hline \multirow{3}{*}{ MPRiB2 } & Ap & $0-8$ & 7.69 & 0.08 & 0.66 & 1.87 \\
\hline & Bw1 & $8-20$ & 7.99 & 0.11 & 0.37 & 2.91 \\
\hline & Bw2 & $20-40$ & 8.01 & 0.29 & 0.22 & 3.08 \\
\hline SWA & & & 7.89 & 0.16 & 0.41 & 2.38 \\
\hline \multicolumn{7}{|c|}{ Gadikusanur series } \\
\hline \multirow[t]{3}{*}{ GKRmB2G1P1 } & Ap & $0-9$ & 7.2 & 0.12 & 0.66 & 8.80 \\
\hline & $\mathrm{Bw}$ & $9-30$ & 7.03 & 0.11 & 0.51 & 9.60 \\
\hline & $\mathrm{Bt}$ & $30-50$ & 7.22 & 0.09 & 0.4 & 10.23 \\
\hline SWA & & & 7.15 & 0.10 & 0.52 & 9.54 \\
\hline \multicolumn{7}{|c|}{ Pashapur series } \\
\hline \multirow{3}{*}{ PPRmB2 } & Ap & $0-9$ & 8.03 & 0.10 & 0.73 & 7.25 \\
\hline & Bw1 & $9-30$ & 8.18 & 0.23 & 0.65 & 8.50 \\
\hline & $\mathrm{Bw} 2$ & $30-70$ & 8.05 & 0.24 & 0.45 & 8.75 \\
\hline SWA & & & 8.08 & 0.19 & 0.61 & 8.16 \\
\hline \multicolumn{7}{|c|}{ DhupatMahagaon series } \\
\hline \multirow[t]{4}{*}{ DMNmB2G1 } & Ap & $0-8$ & 6.56 & 0.08 & 0.79 & 9.15 \\
\hline & $\mathrm{Bw}$ & $8-20$ & 6.49 & 0.05 & 0.57 & 9.48 \\
\hline & Bss1 & $20-50$ & 6.51 & 0.16 & 0.46 & 9.71 \\
\hline & Bss2 & $50-80$ & 6.68 & 0.14 & 0.31 & 10.54 \\
\hline SWA & & & 6.56 & 0.10 & 0.53 & 9.72 \\
\hline \multicolumn{7}{|c|}{ Udawah series } \\
\hline \multirow[t]{5}{*}{ UDHiB2 } & Ap & $0-8$ & 6.47 & 0.07 & 0.74 & 10.15 \\
\hline & $\mathrm{Bw}$ & $8-28$ & 6.69 & 0.11 & 0.66 & 10.90 \\
\hline & Bss1 & $28-50$ & 6.93 & 0.22 & 0.55 & 11.35 \\
\hline & Bss2 & $50-85$ & 7.04 & 0.26 & 0.29 & 11.75 \\
\hline & Bss3 & $85-110$ & 7.65 & 0.29 & 0.18 & 12.75 \\
\hline SWA & & & 6.96 & 0.19 & 0.48 & 11.38 \\
\hline \multicolumn{7}{|c|}{ Kouta- B series } \\
\hline \multirow[t]{5}{*}{ KBBmB2 } & Ap & $0-10$ & 7.86 & 0.12 & 0.87 & 9.75 \\
\hline & $\mathrm{Bw}$ & $10-30$ & 7.98 & 0.14 & 0.83 & 9.95 \\
\hline & Bss1 & $30-70$ & 8.80 & 0.21 & 0.74 & 10.75 \\
\hline & Bss2 & $70-110$ & 8.89 & 0.26 & 0.71 & 10.85 \\
\hline & Bss3 & $110-140$ & 8.90 & 0.28 & 0.69 & 10.91 \\
\hline SWA & & & 8.40 & 0.18 & 0.78 & 10.32 \\
\hline \multicolumn{7}{|c|}{ Kaudgaon series } \\
\hline \multirow[t]{6}{*}{ KGNmB2 } & $\mathrm{Ap}$ & $0-6$ & 8.08 & 0.15 & 0.78 & 9.70 \\
\hline & Bw1 & $6-28$ & 8.23 & 0.17 & 0.74 & 10.14 \\
\hline & Bss1 & $28-70$ & 8.22 & 0.13 & 0.67 & 10.78 \\
\hline & Bss2 & $70-100$ & 8.28 & 0.19 & 0.59 & 11.25 \\
\hline & Bss3 & $100-125$ & 8.15 & 0.21 & 0.45 & 10.98 \\
\hline & Bss4 & $125-150$ & 8.1 & 0.2 & 0.24 & 10.01 \\
\hline SWA & & & 8.17 & 0.17 & 0.57 & 10.47 \\
\hline \multicolumn{7}{|c|}{ Raipalli series } \\
\hline \multirow[t]{6}{*}{ RPLmB2 } & Ap & $0-8$ & 7.95 & 0.06 & 0.78 & 7.65 \\
\hline & $\mathrm{Bw}$ & $8-42$ & 8.46 & 0.23 & 0.69 & 8.00 \\
\hline & Bss1 & $42-60$ & 8.48 & 0.25 & 0.56 & 8.25 \\
\hline & Bss2 & $60-80$ & 8.49 & 0.24 & 0.48 & 9.87 \\
\hline & Bss3 & $80-100$ & 8.50 & 0.26 & 0.44 & 10.12 \\
\hline & Bss4 & $100-150+$ & 8.43 & 0.14 & 0.37 & 10.45 \\
\hline SWA & & & 8.38 & 0.19 & 0.55 & 9.05 \\
\hline
\end{tabular}

Note: $\mathrm{pH}$ - Pouviour of Hydrogen (power of hydrogen), EC- Electrical conductivity, OC- Organic carbon, $\mathrm{CaCO}_{3^{-}}$ Calcium carbonate 
Table.2 Exchangeable cations and cation exchange capacity of the series of Humnabadsub-watershed

\begin{tabular}{|c|c|c|c|c|c|c|c|c|}
\hline \multirow[t]{2}{*}{ Mapping unit } & \multirow[t]{2}{*}{ Horizon } & \multirow[t]{2}{*}{ Depth $(\mathrm{cm})$} & $\mathrm{Ca}^{2+}$ & $\mathrm{Mg}^{2+}$ & $\mathrm{Na}^{+}$ & $\mathrm{K}^{+}$ & CEC & \multirow{2}{*}{$\begin{array}{l}\text { ESP } \\
(\%)\end{array}$} \\
\hline & & & \multicolumn{5}{|c|}{$\operatorname{cmol}\left(\mathrm{p}^{+}\right) \mathrm{kg}^{-1}$} & \\
\hline \multicolumn{9}{|c|}{ Lateritic soil series } \\
\hline \multicolumn{9}{|c|}{ Bhadrapur series } \\
\hline \multirow[t]{2}{*}{ BDPiC3G1 } & Ap & $0-6$ & 8.32 & 3.49 & 0.61 & 0.69 & 25.89 & 2.36 \\
\hline & $\mathrm{Bw}$ & $6-20$ & 8.61 & 3.47 & 0.52 & 0.66 & 26.14 & 1.99 \\
\hline SWA & & & 8.47 & 3.48 & 0.56 & 0.67 & 26.01 & 2.17 \\
\hline \multicolumn{9}{|c|}{ Ramapur series } \\
\hline \multirow[t]{2}{*}{ RMPiC3G2P1 } & Ap & $0-6$ & 8.9 & 3.45 & 0.43 & 0.58 & 27.56 & 1.56 \\
\hline & $\mathrm{Bw}$ & $6-20$ & 8.56 & 3.47 & 0.33 & 0.60 & 28.10 & 1.17 \\
\hline SWA & & & 8.73 & 3.47 & 0.38 & 0.59 & 27.83 & 1.37 \\
\hline \multicolumn{9}{|c|}{ Kadambal series } \\
\hline \multirow[t]{3}{*}{ KDMiB2G2P1 } & Ap & $0-8$ & 8.51 & 2.25 & 0.79 & 0.45 & 24.31 & 3.25 \\
\hline & Bt1 & $8-28$ & 8.38 & 2.06 & 0.84 & 0.61 & 25.64 & 3.28 \\
\hline & $\mathrm{Bt} 2$ & $28-40$ & 8.64 & 2.85 & 0.95 & 0.67 & 25.78 & 3.69 \\
\hline SWA & & & 8.51 & 2.39 & 0.86 & 0.58 & 25.24 & 3.40 \\
\hline \multicolumn{9}{|c|}{ Madargi series } \\
\hline \multirow[t]{3}{*}{ MDGiB2G1 } & Ap & $0-10$ & 8.31 & 2.80 & 1.03 & 0.63 & 25.31 & 4.07 \\
\hline & Bt1 & $10-30$ & 8.20 & 2.15 & 1.21 & 0.58 & 26.40 & 4.58 \\
\hline & $\mathrm{Bt} 2$ & $30-50$ & 8.40 & 2.20 & 0.88 & 0.63 & 26.75 & 3.29 \\
\hline SWA & & & 8.30 & 2.38 & 1.04 & 0.61 & 26.15 & 3.98 \\
\hline \multicolumn{9}{|l|}{ Musthari series } \\
\hline \multirow[t]{3}{*}{ MSTiB2G1P1S1 } & Ap & $0-9$ & 12.84 & 4.17 & 1.25 & 0.76 & 27.36 & 4.57 \\
\hline & Bw1 & $9-30$ & 11.98 & 4.68 & 1.17 & 0.82 & 27.61 & 4.24 \\
\hline & Bw2 & $30-60$ & 13.38 & 4.54 & 0.73 & 0.79 & 28.90 & 2.53 \\
\hline SWA & & & 12.73 & 4.46 & 1.05 & 0.79 & 27.95 & 3.78 \\
\hline \multicolumn{9}{|c|}{ Karanja Khurd series } \\
\hline \multirow[t]{3}{*}{ KKUiB2G2P2 } & Ap & $0-10$ & 13.84 & 5.49 & 0.25 & 0.76 & 28.12 & 0.89 \\
\hline & Bw1 & $10-30$ & 11.80 & 5.14 & 0.95 & 0.69 & 29.17 & 3.26 \\
\hline & Bw2 & $30-70$ & 13.30 & 5.36 & 0.56 & 0.67 & 29.65 & 1.89 \\
\hline SWA & & & 12.98 & 5.33 & 0.58 & 0.71 & 28.98 & 2.01 \\
\hline Muthangi series & & & & & & & & \\
\hline MTNiB2G1 & Ap & $0-9$ & 9.21 & 3.89 & 1.38 & 0.67 & 26.32 & 5.24 \\
\hline & $\mathrm{Bw}$ & $9-25$ & 9.53 & 3.95 & 1.37 & 0.66 & 28.71 & 4.77 \\
\hline & Bt1 & $25-60$ & 9.77 & 4.35 & 1.07 & 0.68 & 29.50 & 3.63 \\
\hline & Bt3 & $60-98$ & 9.39 & 4.65 & 1.02 & 0.79 & 30.25 & 3.37 \\
\hline SWA & & & 9.48 & 4.21 & 1.21 & 0.70 & 28.69 & 4.00 \\
\hline & & & i series & & & & & \\
\hline DGRiB2G1P1 & Ap & $0-8$ & 13.04 & 5.47 & 1.40 & 0.85 & 24.78 & 5.65 \\
\hline & Bw1 & $8-30$ & 13.38 & 8.24 & 1.18 & 0.80 & 25.41 & 4.64 \\
\hline & Bw2 & $30-60$ & 13.55 & 7.96 & 0.87 & 0.65 & 26.56 & 3.28 \\
\hline & $\mathrm{Bw} 3$ & $60-85$ & 13.42 & 8.25 & 0.86 & 0.72 & 27.45 & 3.13 \\
\hline & Bw4 & $85-120$ & 13.09 & 8.59 & 0.79 & 0.78 & 27.36 & 2.89 \\
\hline SWA & & & 13.29 & 7.70 & 1.02 & 0.76 & 26.05 & 3.92 \\
\hline & & $\mathrm{Mu}$ & adi ser & & & & & \\
\hline MWDiB2 & Ap & $0-8$ & 8.58 & 3.40 & 0.80 & 0.61 & 25.67 & 3.12 \\
\hline & $\mathrm{Bw}$ & $88-39$ & 8.41 & 3.48 & 0.78 & 0.60 & 25.98 & 3.00 \\
\hline & Bt1 & $39-76$ & 8.29 & 3.40 & 0.75 & 0.77 & 26.51 & 2.83 \\
\hline & $\mathrm{Bt} 2$ & $76-98$ & 8.04 & 3.27 & 0.76 & 0.58 & 27.45 & 2.77 \\
\hline & Bt3 & $98-140$ & 7.57 & 3.34 & 1.03 & 0.59 & 27.96 & 3.68 \\
\hline & $\mathrm{Bt} 4$ & $140+$ & 7.03 & 3.30 & 1.12 & 0.59 & 28.10 & 3.99 \\
\hline SWA & & & 7.99 & 3.36 & 0.87 & 0.62 & 26.40 & 3.23 \\
\hline & & Bas & oil ser & & & & & \\
\hline
\end{tabular}




\begin{tabular}{|c|c|c|c|c|c|c|c|c|}
\hline \multicolumn{9}{|l|}{ Lingi series } \\
\hline \multirow[t]{2}{*}{ LINiD3G2P2S4 } & Ap & $0-10$ & 18.59 & 7.26 & 3.90 & 0.63 & 38.90 & 10.03 \\
\hline & $\mathrm{Bw}$ & $10-21$ & 18.61 & 7.30 & 3.72 & 0.58 & 39.54 & 9.41 \\
\hline SWA & & & 18.6 & 7.28 & 3.58 & 0.61 & 39.22 & 9.72 \\
\hline \multicolumn{9}{|l|}{ Mustapur series } \\
\hline \multirow[t]{3}{*}{ MPRiB2 } & Ap & $0-8$ & 13.65 & 5.69 & 0.81 & 0.74 & 24.87 & 3.26 \\
\hline & Bw1 & $8-20$ & 12.98 & 5.93 & 0.75 & 0.66 & 25.41 & 2.95 \\
\hline & Bw2 & $20-40$ & 10.14 & 5.83 & 0.65 & 0.63 & 25.98 & 2.50 \\
\hline SWA & & & 12.26 & 5.82 & 0.73 & 0.67 & 25.42 & 2.90 \\
\hline \multicolumn{9}{|c|}{ Gadikusanur series } \\
\hline \multirow[t]{3}{*}{ GKRmB2G1P1 } & Ap & $0-9$ & 29.43 & 8.19 & 3.97 & 0.75 & 40.10 & 9.90 \\
\hline & $\mathrm{Bw}$ & $9-30$ & 29.08 & 7.38 & 3.69 & 0.63 & 40.25 & 9.17 \\
\hline & $\mathrm{Bt}$ & $30-50$ & 28.9 & 7.78 & 3.16 & 0.64 & 40.98 & 7.71 \\
\hline SWA & & & 29.14 & 7.78 & 3.61 & 0.67 & 40.44 & 8.93 \\
\hline \multicolumn{9}{|l|}{ Pashapur series } \\
\hline \multirow[t]{3}{*}{ PPRmB2 } & Ap & $0-9$ & 21.81 & 4.97 & 3.80 & 0.61 & 32.36 & 11.74 \\
\hline & Bw1 & $9-30$ & 21.76 & 4.69 & 3.97 & 0.62 & 33.50 & 11.85 \\
\hline & Bw2 & $30-70$ & 21.04 & 4.45 & 3.72 & 0.61 & 33.87 & 10.98 \\
\hline SWA & & & 21.54 & 4.71 & 3.83 & 0.61 & 33.24 & 11.53 \\
\hline
\end{tabular}

\begin{tabular}{|c|c|c|c|c|c|c|c|c|}
\hline \multicolumn{9}{|c|}{ DhupatMahagaon series } \\
\hline \multirow[t]{4}{*}{ DMNmB2G1 } & Ap & $0-8$ & 27.48 & 8.11 & 1.06 & 0.84 & 38.94 & 2.72 \\
\hline & $\mathrm{Bw}$ & $8-20$ & 27.35 & 7.85 & 1.03 & 0.66 & 39.12 & 2.63 \\
\hline & Bss1 & $20-50$ & 27.47 & 7.58 & 0.35 & 0.81 & 40.20 & 0.87 \\
\hline & Bss2 & $50-80$ & 27.78 & 7.95 & 0.41 & 0.82 & 41.23 & 0.99 \\
\hline SWA & & & 27.52 & 7.87 & 0.71 & 0.78 & 39.87 & 1.81 \\
\hline \multicolumn{9}{|c|}{ Udawah series } \\
\hline \multirow{5}{*}{ UDHmB2 } & Ap & $0-8$ & 22.51 & 8.63 & 1.13 & 0.58 & 34.16 & 3.31 \\
\hline & $\mathrm{Bw}$ & $8-28$ & 22.94 & 8.62 & 0.79 & 0.62 & 35.84 & 2.20 \\
\hline & Bss1 & $28-50$ & 22.9 & 8.62 & 0.75 & 0.85 & 36.12 & 2.08 \\
\hline & Bss2 & $50-85$ & 23.96 & 8.60 & 0.63 & 0.68 & 38.79 & 1.62 \\
\hline & Bss3 & $85-110$ & 23.02 & 8.50 & 0.61 & 0.68 & 38.09 & 1.60 \\
\hline SWA & & & 23.07 & 8.59 & 0.78 & 0.68 & 36.60 & 2.16 \\
\hline \multicolumn{9}{|l|}{ Kouta- B series } \\
\hline \multirow[t]{5}{*}{$\mathrm{KBBmB} 2$} & Ap & $0-10$ & 13.36 & 8.56 & 2.50 & 0.64 & 34.20 & 7.31 \\
\hline & $\mathrm{Bw}$ & $10-30$ & 13.49 & 9.98 & 2.48 & 0.63 & 35.64 & 6.96 \\
\hline & Bss1 & $30-70$ & 13.63 & 9.75 & 3.07 & 0.64 & 36.24 & 8.47 \\
\hline & Bss2 & $70-110$ & 14.25 & 8.46 & 3.10 & 0.65 & 36.57 & 8.48 \\
\hline & Bss3 & $110-140$ & 14.31 & 8.48 & 3.24 & 0.68 & 36.78 & 8.52 \\
\hline SWA & & & 13.68 & 9.19 & 2.79 & 0.64 & 35.66 & 7.80 \\
\hline \multicolumn{9}{|c|}{ Kaudgaon series } \\
\hline \multirow[t]{6}{*}{ KGNmB2 } & Ap & $0-6$ & 28.32 & 8.47 & 3.53 & 0.70 & 39.65 & 8.90 \\
\hline & Bw1 & $6-28$ & 29.03 & 9.15 & 3.46 & 0.60 & 39.97 & 8.66 \\
\hline & Bss1 & $28-70$ & 28.98 & 9.94 & 2.60 & 0.70 & 40.23 & 6.46 \\
\hline & Bss2 & $70-100$ & 29.12 & 8.42 & 2.51 & 0.77 & 41.56 & 6.04 \\
\hline & Bss3 & $100-125$ & 29.92 & 8.51 & 2.14 & 0.74 & 41.85 & 5.11 \\
\hline & Bss4 & $125-150$ & 28.70 & 8.43 & 2.02 & 0.66 & 40.10 & 5.04 \\
\hline SWA & & & 28.85 & 8.82 & 2.71 & 0.70 & 40.56 & 6.70 \\
\hline \multicolumn{9}{|c|}{ Raipalli series } \\
\hline \multirow[t]{6}{*}{ RPLmB2 } & Ap & $0-8$ & 22.82 & 8.99 & 3.75 & 0.86 & 35.02 & 12.49 \\
\hline & $\mathrm{Bw}$ & $8-42$ & 23.86 & 8.96 & 3.40 & 0.77 & 36.47 & 10.80 \\
\hline & Bss1 & $42-60$ & 23.90 & 8.98 & 3.31 & 0.72 & 36.98 & 10.04 \\
\hline & Bss2 & $60-80$ & 23.97 & 8.98 & 3.29 & 0.73 & 36.46 & 9.55 \\
\hline & Bss3 & $80-100$ & 23.66 & 9.04 & 3.24 & 0.74 & 37.05 & 9.24 \\
\hline & Bss4 & $100-150+$ & 23.14 & 9.02 & 2.09 & 0.74 & 37.16 & 5.94 \\
\hline SWA & & & 23.56 & 8.99 & 3.18 & 0.76 & 36.52 & 9.68 \\
\hline
\end{tabular}

Note: CEC- Cation exchange capacity, ESP- Exchangeable sodium percentage 
Table.3 Fertility status of the soil series of Humnabad sub-watershed

\begin{tabular}{|c|c|c|c|c|c|c|c|c|c|c|}
\hline \multirow[t]{3}{*}{ Mapping units } & \multirow[t]{3}{*}{ Horizons } & \multirow[t]{3}{*}{ Depth $(\mathrm{cm})$} & \multicolumn{4}{|c|}{ Available nutrients } & \multicolumn{4}{|c|}{ DTPA extractable } \\
\hline & & & $\mathbf{N}$ & $\mathrm{P}_{2} \mathrm{O}_{5}$ & $\mathrm{~K}_{2} \mathrm{O}$ & \multirow{2}{*}{$\begin{array}{l}\text { S } \\
\text { ppm }\end{array}$} & $\mathrm{Cu}$ & Zn & $\mathrm{Fe}$ & Mn \\
\hline & & & \multicolumn{3}{|c|}{ kg ha $^{-1}$} & & \multicolumn{4}{|c|}{ ppm } \\
\hline \multicolumn{11}{|c|}{ Lateritic soil series } \\
\hline \multicolumn{11}{|c|}{ Bharapur series } \\
\hline \multirow[t]{2}{*}{ BDPiC3G1 } & Ap & $0-6$ & 263.42 & 10.71 & 269.70 & 12.42 & 3.34 & 0.84 & 7.12 & 3.76 \\
\hline & $\mathrm{Bw}$ & $6-20$ & 250.88 & 11.53 & 256.82 & 13.45 & 3.58 & 1.24 & 5.40 & 3.66 \\
\hline SWA & & & 257.15 & 11.12 & 263.26 & 12.94 & 3.46 & 1.04 & 6.26 & 3.71 \\
\hline \multicolumn{11}{|l|}{ Ramapur series } \\
\hline \multirow[t]{2}{*}{ RMPiC3G2P1 } & Ap & $0-6$ & 263.42 & 7.41 & 225.68 & 18.02 & 1.23 & 0.61 & 6.80 & 3.66 \\
\hline & $\mathrm{Bw}$ & $6-20$ & 238.33 & 9.05 & 233.96 & 15.78 & 1.42 & 0.58 & 8.00 & 3.26 \\
\hline SWA & & & 250.88 & 8.23 & 229.82 & 16.90 & 1.33 & 0.60 & 7.40 & 3.46 \\
\hline \multicolumn{11}{|c|}{ Kadambalseries } \\
\hline \multirow[t]{3}{*}{ KDMiB2G2P1 } & $\mathrm{Ap}$ & $0-8$ & 238.33 & 25.52 & 177.18 & 17.9 & 4.94 & 0.12 & 7.18 & 3.69 \\
\hline & Bt1 & $8-28$ & 213.24 & 2.47 & 239.56 & 19.17 & 3.28 & 0.38 & 6.15 & 3.25 \\
\hline & $\mathrm{Bt} 2$ & $28-60$ & 188.16 & 1.64 & 260.73 & 19.54 & 4.80 & 0.22 & 4.41 & 2.94 \\
\hline SWA & & & 213.24 & 9.88 & 225.82 & 18.87 & 4.34 & 0.24 & 5.91 & 3.29 \\
\hline \multicolumn{11}{|c|}{ Madargi series } \\
\hline \multirow[t]{3}{*}{ MDGiB2G1 } & Ap & $0-10$ & 291.23 & 21.41 & 246.96 & 15.20 & 3.64 & 1.47 & 8.00 & 3.74 \\
\hline & Bt1 & $10-30$ & 288.69 & 17.29 & 225.12 & 15.23 & 3.4 & 1.26 & 8.06 & 3.72 \\
\hline & $\mathrm{Bt} 2$ & $30-50$ & 271.06 & 12.35 & 246.18 & 11.00 & 3.02 & 2.74 & 7.08 & 3.83 \\
\hline SWA & & & 283.66 & 17.02 & 239.42 & 13.81 & 3.35 & 1.82 & 7.71 & 3.76 \\
\hline & & & Mustl & series & & & & & & \\
\hline MSTiB2G1P1S1 & Ap & $0-9$ & 263.424 & 22.79 & 255.57 & 14.23 & 4.36 & 1.46 & 9.23 & 3.96 \\
\hline & Bw1 & $9-30$ & 263.424 & 20.13 & 270.99 & 12.50 & 3.81 & 1.32 & 8.29 & 3.34 \\
\hline & Bw2 & $30-60$ & 275.968 & 17.29 & 298.46 & 8.96 & 2.11 & 0.93 & 2.57 & 3.02 \\
\hline SWA & & & 267.60 & 20.07 & 275.01 & 13.36 & 3.43 & 1.24 & 6.70 & 3.44 \\
\hline & & & Karanja & urd seri & & & & & & \\
\hline KKUiB2G2P2 & Ap & $0-10$ & 263.42 & 23.05 & 298.70 & 17.93 & 3.39 & 0.44 & 6.95 & 3.28 \\
\hline & Bw1 & $10-30$ & 238.34 & 22.23 & 270.48 & 14.57 & 3.54 & 0.68 & 4.89 & 3.66 \\
\hline & Bw2 & $30-70$ & 238.34 & 17.29 & 261.97 & 10.08 & 3.05 & 0.62 & 3.98 & 3.76 \\
\hline SWA & & & 246.70 & 20.86 & 277.05 & 14.19 & 3.33 & 0.58 & 5.27 & 3.57 \\
\hline & & & Mutha & i series & & & & & & \\
\hline MTNiB2G1 & Ap & $0-9$ & 338.688 & 27.99 & 261.52 & 25.78 & 2.47 & 1.34 & 7.24 & 2.38 \\
\hline & $\mathrm{Bw}$ & $9-25$ & 263.424 & 13.98 & 258.72 & 15.69 & 1.38 & 0.48 & 6.33 & 2.60 \\
\hline & Bt1 & $25-60$ & 213.248 & 10.7 & 266.33 & 8.98 & 2.91 & 0.52 & 5.16 & 3.13 \\
\hline & Bt3 & $60-98$ & 188.16 & 8.23 & 309.68 & 7.90 & 3.39 & 0.68 & 5.04 & 2.34 \\
\hline SWA & & & 250.88 & 15.22 & 274.06 & 14.58 & 2.53 & 0.75 & 5.94 & 2.61 \\
\hline & & & Dev & series & & & & & & \\
\hline DGRiB2G1P1 & Ap & $0-8$ & 288.51 & 15.64 & 331.29 & 20.17 & 4.70 & 0.92 & 8.90 & 1.15 \\
\hline & Bw1 & $8-30$ & 163.07 & 14.82 & 312.70 & 12.51 & 4.18 & 0.97 & 7.42 & 2.03 \\
\hline & Bw2 & $30-60$ & 213.24 & 10.7 & 253.0 & 19.14 & 2.87 & 0.64 & 3.00 & 2.22 \\
\hline & Bw3 & $60-85$ & 163.72 & 9.88 & 281.56 & 19.05 & 2.06 & 0.42 & 1.86 & 2.99 \\
\hline & Bw4 & $85-120$ & 175.61 & 9.05 & 303.18 & 16.9 & 1.59 & 0.54 & 1.79 & 2.76 \\
\hline SWA & & & 200.70 & 12.01 & 296.35 & 17.55 & 3.08 & 0.70 & 4.59 & 2.23 \\
\hline & & & Musta & di series & & & & & & \\
\hline MWDiB2 & Ap & $0-8$ & 313.6 & 38.9 & 236.99 & 17.93 & 1.54 & 0.52 & 7.48 & 2.88 \\
\hline & $\mathrm{Bw}$ & $8-39$ & 288.51 & 23.05 & 234.52 & 18.17 & 1.05 & 0.49 & 8.36 & 2.64 \\
\hline & Bt1 & $39-76$ & 273.51 & 13.17 & 302.51 & 19.78 & 1.54 & 0.52 & 8.18 & 3.74 \\
\hline & $\mathrm{Bt} 2$ & $76-98$ & 250.88 & 10.7 & 225.79 & 19.17 & 1.72 & 0.49 & 8.98 & 3.28 \\
\hline & Bt3 & $98-140$ & 238.33 & 9.88 & 232.51 & 15.69 & 1.98 & 0.72 & 7.38 & 3.21 \\
\hline & Bt4 & $140+$ & 200.70 & 9.05 & 231.72 & 10.08 & 2.04 & 0.68 & 6.34 & 3.10 \\
\hline SWA & & & 263.42 & 17.45 & 244.01 & 16.80 & 1.65 & 0.57 & 7.79 & 3.14 \\
\hline & & & Basalt & il serie & & & & & & \\
\hline
\end{tabular}


Int.J.Curr.Microbiol.App.Sci (2019) 8(2): $3264-3273$

\begin{tabular}{|c|c|c|c|c|c|c|c|c|c|c|}
\hline \multicolumn{11}{|c|}{ Lingi Series } \\
\hline \multirow{2}{*}{ LINiD3G2P2S4 } & Ap & $0-10$ & 200.70 & 11.53 & 244.83 & 17.93 & 2.25 & 1.04 & 3.19 & 3.08 \\
\hline & $\mathrm{Bw}$ & $10-21$ & 150.53 & 8.24 & 228.59 & 11.21 & 1.67 & 0.74 & 3.16 & 3.28 \\
\hline SWA & & & 175.62 & 9.88 & 236.71 & 14.57 & 1.96 & 0.89 & 3.17 & 3.18 \\
\hline \multicolumn{11}{|c|}{ Mustapur series } \\
\hline \multirow[t]{3}{*}{ MPRiB2 } & Ap & $0-8$ & 263.42 & 14.00 & 288.62 & 17.93 & 3.45 & 0.98 & 9.08 & 2.62 \\
\hline & Bw1 & $8-20$ & 250.88 & 9.88 & 256.26 & 13.45 & 2.60 & 0.62 & 5.16 & 3.56 \\
\hline & Bw2 & $20-40$ & 200.70 & 8.24 & 245.28 & 12.33 & 2.72 & 0.71 & 6.14 & 2.04 \\
\hline SWA & & & 238.34 & 10.71 & 263.39 & 14.57 & 2.92 & 0.77 & 6.79 & 2.74 \\
\hline \multicolumn{11}{|c|}{ Gadikusanur series } \\
\hline \multirow[t]{3}{*}{ GKRmB2G1P1 } & Ap & $0-9$ & 275.97 & 27.17 & 292.54 & 8.96 & 1.49 & 0.79 & 3.64 & 2.88 \\
\hline & $\mathrm{Bw}$ & $9-30$ & 263.42 & 13.99 & 246.85 & 15.69 & 1.37 & 1.12 & 3.74 & 2.64 \\
\hline & $\mathrm{Bt}$ & $30-50$ & 238.34 & 9.05 & 251.55 & 17.93 & 1.10 & 1.01 & 2.43 & 1.78 \\
\hline SWA & & & 259.24 & 16.74 & 263.65 & 14.19 & 1.32 & 0.97 & 3.27 & 2.43 \\
\hline \multicolumn{11}{|c|}{ Pashapur series } \\
\hline \multirow[t]{3}{*}{ PPRmB2 } & Ap & $0-9$ & 301.056 & 26.23 & 239.00 & 15.69 & 2.18 & 0.52 & 1.36 & 2.55 \\
\hline & Bw1 & $9-30$ & 275.968 & 14.82 & 240.91 & 17.66 & 3.78 & 0.88 & 1.89 & 2.18 \\
\hline & Bw2 & $30-70$ & 225.792 & 12.35 & 238.89 & 18.2 & 0.64 & 0.51 & 0.95 & 2.35 \\
\hline SWA & & & 267.60 & 17.80 & 239.60 & 17.18 & 2.20 & 0.64 & 1.40 & 2.36 \\
\hline \multicolumn{11}{|c|}{ DhupatMahagaon series } \\
\hline \multirow[t]{4}{*}{ DMNmB2G1 } & Ap & $0-8$ & 288.51 & 23.05 & 327.26 & 20.17 & 3.64 & 1.32 & 4.05 & 2.05 \\
\hline & $\mathrm{Bw}$ & $8-20$ & 263.42 & 18.94 & 257.60 & 17.90 & 4.04 & 1.04 & 8.52 & 0.54 \\
\hline & Bss1 & $20-50$ & 225.79 & 10.70 & 318.19 & 18.42 & 3.99 & 1.92 & 8.19 & 0.44 \\
\hline & Bss2 & $50-80$ & 188.16 & 7.41 & 319.65 & 18.66 & 3.50 & 1.04 & 8.20 & 0.48 \\
\hline SWA & & & 241.47 & 15.03 & 305.68 & 18.79 & 3.79 & 1.33 & 7.24 & 0.88 \\
\hline \multicolumn{11}{|c|}{ Udawah series } \\
\hline \multirow[t]{5}{*}{ UDHiB2 } & Ap & $0-8$ & 338.69 & 21.71 & 228.03 & 12.33 & 3.04 & 0.92 & 3.54 & 1.27 \\
\hline & $\mathrm{Bw}$ & $8-28$ & 275.97 & 21.58 & 242.59 & 7.84 & 3.67 & 0.82 & 4.93 & 0.50 \\
\hline & Bss1 & $28-50$ & 250.88 & 20.88 & 331.63 & 6.72 & 3.18 & 0.48 & 5.13 & 2.07 \\
\hline & Bss2 & $50-85$ & 225.79 & 20.24 & 267.12 & 16.81 & 1.47 & 0.43 & 2.62 & 0.98 \\
\hline & Bss3 & $85-110$ & 188.16 & 20.01 & 265.22 & 11.21 & 1.83 & 0.42 & 2.11 & 1.34 \\
\hline SWA & & & 255.90 & 20.88 & 266.92 & 10.98 & 2.64 & 0.61 & 3.67 & 1.23 \\
\hline \multicolumn{11}{|c|}{ Kouta- B series } \\
\hline \multirow[t]{5}{*}{$\mathrm{KBBmB2}$} & Ap & $0-10$ & 288.16 & 21.52 & 250.65 & 23.54 & 3.24 & 0.81 & 2.71 & 1.81 \\
\hline & $\mathrm{Bw}$ & $10-30$ & 263.42 & 21.17 & 244.83 & 17.93 & 4.42 & 1.70 & 1.14 & 2.36 \\
\hline & Bss1 & $30-70$ & 213.24 & 20.08 & 251.82 & 11.30 & 5.54 & 2.14 & 1.24 & 1.34 \\
\hline & Bss2 & $70-110$ & 175.61 & 20.06 & 254.51 & 7.84 & 1.68 & 0.66 & 1.29 & 1.25 \\
\hline & Bss3 & $110-140$ & 175.04 & 20.01 & 253.05 & 7.32 & 1.23 & 0.42 & 1.02 & 1.13 \\
\hline SWA & & & 235.10 & 20.71 & 250.45 & 15.15 & 3.72 & 1.33 & 1.60 & 1.69 \\
\hline \multicolumn{11}{|c|}{ Kaudgaon series } \\
\hline \multirow[t]{6}{*}{ KGNmB2 } & Ap & $0-6$ & 315.97 & 27.88 & 273.17 & 16.81 & 3.39 & 0.98 & 3.24 & 1.92 \\
\hline & Bw1 & $6-28$ & 309.42 & 27.05 & 234.53 & 15.69 & 4.00 & 1.07 & 2.18 & 1.90 \\
\hline & Bss1 & $28-70$ & 302.25 & 26.55 & 272.50 & 19.05 & 3.34 & 0.98 & 1.29 & 1.02 \\
\hline & Bss2 & $70-100$ & 297.62 & 26.23 & 301.95 & 18.42 & 3.91 & 1.05 & 1.27 & 1.20 \\
\hline & Bss3 & $100-125$ & 284.62 & 25.41 & 290.53 & 17.93 & 3.94 & 0.76 & 1.16 & 1.13 \\
\hline & Bss4 & $125-150$ & 277.07 & 24.58 & 259.50 & 12.30 & 2.86 & 0.55 & 1.02 & 1.23 \\
\hline SWA & & & 297.82 & 26.28 & 272.03 & 16.70 & 3.57 & 0.90 & 1.69 & 1.40 \\
\hline & & & Raip & series & & & & & & \\
\hline RPLmB2 & Ap & $0-8$ & 358.51 & 28.71 & 335.66 & 17.93 & 1.62 & 0.64 & 1.57 & 4.72 \\
\hline & $\mathrm{Bw}$ & $8-42$ & 330.88 & 18.18 & 302.62 & 13.45 & 1.37 & 0.66 & 1.16 & 3.78 \\
\hline & Bss1 & $42-60$ & 313.07 & 28.06 & 280.00 & 5.60 & 2.59 & 0.58 & 1.42 & 3.98 \\
\hline & Bss2 & $60-80$ & 303.07 & 27.18 & 284.14 & 4.48 & 1.03 & 0.73 & 1.12 & 3.56 \\
\hline & Bss3 & $80-100$ & 295.44 & 26.53 & 287.39 & 12.33 & 2.30 & 0.66 & 1.90 & 3.40 \\
\hline & Bss4 & $100150+$ & 270.35 & 26.18 & 288.28 & 14.57 & 1.48 & 0.54 & 1.18 & 2.42 \\
\hline SWA & & & 311.89 & 25.80 & 296.35 & 11.39 & 1.57 & 0.64 & 1.39 & 3.64 \\
\hline
\end{tabular}


The exchangeable calcium and magnesium ranged from 7.99 to $29.14 \mathrm{cmol}\left(\mathrm{p}^{+}\right) \mathrm{kg}^{-1}$ and 2.38 to $9.19 \mathrm{cmol}\left(\mathrm{p}^{+}\right) \mathrm{kg}^{-1}$, respectively. The cation exchange capacity of various soil pedons ranged from moderate to high. The black soil pedons exhibited higher CEC due to higher clay content and predominance of 2:1 type clay minerals where as in case of red soils it was moderate as shown in Table 2.

Table 3 shows that the available nitrogen status of the watershed ranged from low to medium and varied from (SWA) 175.60 to $330.33 \mathrm{~kg} \mathrm{ha}^{-1}$ in Humnabad sub-watershed. The available phosphorus ranged from low to medium and varied from (SWA) 8.23 to 20.86 $\mathrm{kg} \mathrm{ha}^{-1}$. The available potassium ranged from low to medium and varied from (SWA) 225.82 to $305.68 \mathrm{~kg} \mathrm{ha}^{-1}$. The available sulphur status ranged from low to high but most of the area was low to medium and values varied from (SWA) 18.87 to 10.98 ppm.

The DTPA extractable zinc varied from (SWA) 0.24 to $1.82 \mathrm{ppm}$, copper varied from (SWA) 1.32 to $4.34 \mathrm{ppm}$, manganese varied from (SWA) 0.88 to $3.76 \mathrm{ppm}$ and iron varied from (SWA) 1.35 to $7.79 \mathrm{ppm}$. All the micronutrients were sufficient in lateritic soil series while, in basaltic soil series iron and zinc were low.

The soil reaction of pedons varied from neutral to slightly alkaline. Soil $\mathrm{pH}$ and EC of major soil pedons increased with depth due to accumulation of leached bases in the subsurface horizons. In general, the surface soil horizons recorded higher organic carbon content than underlying layers and decreased with depth in all soil pedons. The free calcium carbonate content increased with depth. The exchangeable bases were in the order of $\mathrm{Ca}^{2+}>\mathrm{Mg}^{2+}>\mathrm{Na}^{+}>\mathrm{K}^{+}$on the exchangeable complex. The cation exchange capacity of various soil pedons ranged from moderate to high. The black soil pedons exhibited higher CEC due to higher clay content and predominance of 2:1 type clay minerals where as in case of red soils it was moderate.

The available nitrogen status of the watershed ranged from low to medium. The available phosphorus and potassium ranged from low to medium. The available sulphur status ranged from low to high but most of the area was low to medium. The present study shows that the study area was sufficient in micronutrients in lateritic soil series while, in basaltic soil series iron and zinc were low.

\section{References}

Bahi, M. R., Vidyavathi, G. Y., Yadahalli, G. S., Rudramurthy, H. V. and Rajesh, N. L., 2017(b), Land resources characterization and soil classification of Pannur North-3 micro-watershed using RS and GIS in Manvi Taluk, Raichur district. J. Pharmacognosy and Phytochemistry, 7(1): 671-678.

Denis, M. K. A., Patil, L. P., Kamara, A. M. and Saidu, D. H., 2017, Assessment of soil fertility status using nutrient index approach. Academia J. Agri. Res, 5(2): 28-38.

Nalina, C. N., Anilkumar, K. S., Shilpashree, K. G., Babu, N., Sudhir, K. and Natarajan, A., 2017, Inventory and mapping of land resources for land use planning through detail soil survey coupled with remote sensing and GIS Techniques: A case study in Nagenahalli watershed, Doddaballapur taluk, Bangalore rural district, India. Int.J.Curr.Microbiol.App.Sci., $\quad$ 6(8): 314-331.

Ravindra Naik, M. and Anil Kumar. K. S., 2015, Fertility status of Kannur microwatershed soils in Chamarajanagar, Karnataka. Int. J. Sci. Res. Pub., 5(9): 1-10. 
Sivasankaran, K., Mithyantha, M. S., Natesan, S. and Subbarayappa, C. T., 1993, Physico-chemical properties and nutrient management of red and lateritic soils under plantation crops in Southern India, NBSS Publication, 37: 280.

\section{How to cite this article:}

Thejaswini C., Ashok S. Alur, M. Shivanna, S. Anil Kumar, S. J. Prashanth, B. N. Dhannanjaya and Praveen Kumar B. Naikodi. 2019. Land Resource Inventory and Soil Mapping for Fertility Status of Humnabad Sub-Watershed. Int.J.Curr.Microbiol.App.Sci. 8(02): 3264-3273. doi: https://doi.org/10.20546/ijcmas.2019.802.381 